\title{
Sputtering Yield of Noble Gas Irradiation onto Tungsten Surface
}

\author{
Hiroaki Nakamura $^{1,2, *}$, Seiki Saito ${ }^{3}$, Atsushi M. Ito ${ }^{1}$ \\ ${ }^{1}$ Department of Helical Plasma Research, National Institute for Fusion Science \\ ${ }^{2}$ Department of Energy Engineering and Science, Nagoya University \\ ${ }^{3}$ Department of Electrical Engineering, National Institute of Technology, Kushiro College \\ *hnakamura@nifs.ac.jp
}

Received: January 30, 2016; Accepted: March 14, 2016; Published: April 7, 2017

\begin{abstract}
It is necessary to evaluate the endurance of tungsten (W) under plasma irradiation to realize the nuclear fusion reactor. The tungsten that has flat surface and amorphous structure is often assumed in the experiment and the simulation in plasma-wall-interaction study. However, from experiments, it has been reported that the sputtering yield for the flat surface $\mathrm{W}$ under argon (Ar) irradiation is different from that for the rough (fuzz) surface W. In our previous work, using binary-collision-approximation (BCA) simulation, we calculated the sputtering yield under Ar irradiation onto W target which has "simplified rough surface." We could obtain the roughness dependence of the sputtering yield, which is coincident qualitatively with the experimental result. In this paper, we also calculated the physical quantities, i.e., sputtering yield, retention rate and mean penetration depth, under the noble (helium, neon, or argon) gas irradiation onto four types of the flat $\mathrm{W}$ targets: Amorphous $\mathrm{W}$ and BCC W lattices with the crystal orientation (110), (100) and (111). This simulation shows that the W surface structure affects noticeably the physical quantities. From these simulations, we propose that it is necessary to consider the surface form and the crystal structure to evaluate the sputtering phenomena though it has not been considered sufficiently.
\end{abstract}

Keywords: Plasma facing material, Binary collision approximation, Tungsten, Crystal Orientation, Amorphous Structure, Noble gases

\section{Introduction}

Fiberform nano-structured tungsten discovered by S. Takamura et al. [1] is called tungsten fuzz structure. This structure is formed when a helium $(\mathrm{He})$ atom is irradiated onto the tungsten (W) surface with appropriate temperature of W and irradiation energy of He. From the viewpoint of plasma confinement in nuclear fusion, the $\mathrm{W}$ fuzz structure is regarded as an obstacle because the fuzz is so fragile that it has the risk of decreasing plasma temperature if the portion of the fuzz is detached from the tungsten wall. In order to avoid the fuzz structure 
formation on the surface of tungsten, much experimental research was performed to obtain the physical quantities of tungsten fuzz structure $[2,3]$. However, the structure formation of fuzz structure is still an unresolved problem. Numerical simulation as well as experimental studies is necessary to reveal this problem. This is the background of our simulation study. We, thus, have been developing binary-collision-approximation (BCA) simulation to reveal the sputtering phenomena between tungsten target and inject atoms [4-7].

In our previous work [8], we investigated the dependence of the sputtering phenomena on the surface roughness, based on the experiment that was performed by Nishijima et al. [3]. According to their work, the sputtering yield depends on the surface roughness, that is, the thickness of the fuzz layer. However, the reason for this dependence is not clear. Therefore, using BCA simulation, we have revealed the dependence on the thickness of the fuzz layer [8].

In this paper, picking up the sputtering phenomena of $\mathrm{W}$ irradiated by noble gases as well as in our previous work, we perform the BCA simulation. In this simulation, we reveal the dependence of the sputtering phenomena on the crystal orientation of the target tungsten. It is usual to simplify the target material by the amorphous structure to treat the sputtering phenomena. This simplification is not appropriate to treat the channeling phenomena of the crystal structure. The channeling effect is as follows: When the target material has mono-crystalline structure, the trajectory of the injected atom in the target depends on the orientation of the atom relative to the crystalline axes. We, therefore, clarify the dependence on the crystal orientation of the tungsten to treat the crystal target structure as well as the amorphous target.

\section{Dependence on Crystalline Structure of Tungsten}

\subsection{Binary Collision Approximation (BCA) Simulation}

We use a binary collision approximation (BCA) to solve scattering phenomena between tungsten atoms and injected noble gas atom. The BCA simulation is performed by AC $\forall \mathrm{T}$ (atomic collision in any structured target) code [4-6]. In the BCA simulation, multi-body interactions in a material approximate to consecutive two-body interactions between a projectile atom and the nearest neighbor atom. Using this code, we calculated the sputtering yield which gives basic information to reveal the nano-structure formation [6]. Please see Refs. 4 and 5 for the details of the BCA simulation. By this simulation, we reveal the dependence of the sputtering phenomena on the crystal orientation of the target tungsten [7].

\subsection{Simulation Model}

The tungsten target in the simulation shown in Fig. 1 is $94.95 \AA$ long, $47.25 \AA$ wide and $31650 \AA$ A deep. Periodic boundary conditions are imposed on the horizontal direction of the tungsten target in Fig. 1. As the lattice structure of the $\mathrm{W}$ target, we prepared four types of the lattice structure: Amorphous structure and BCC lattices with the crystal orientation (110), (100), and (111). In the BCC lattice, all tungsten atoms are fixed to the lattice points of BCC structure without thermal vibration. The lattice constant of tungsten BCC structure is 


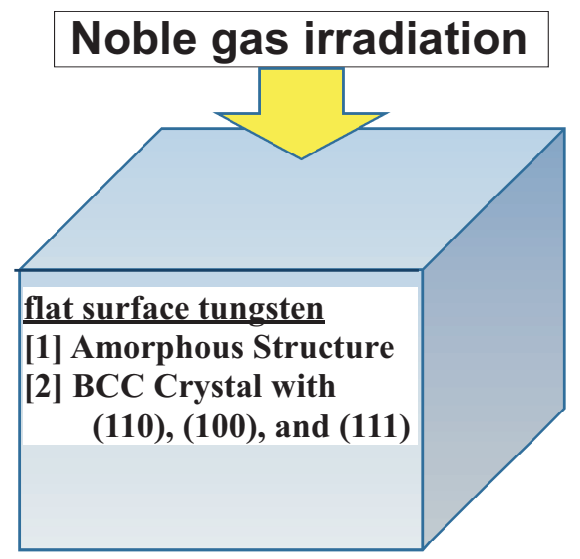

Figure 1: Tungsten target in BCA simulation. The atomic structure of the $\mathrm{W}$ target has the following four types: Amorphous structure, BCC lattices with the crystal orientation (110), (100), and (111). Helium (He), neon (Ne), and argon (Ar) are injected from the top of the target vertically.

$3.165 \AA$. On the other hand, tungsten atoms are distributed randomly in amorphous structure under the condition that the number density of $\mathrm{W}$ is the same as the BCC lattice. Thus, we prepared four types of the $\mathrm{W}$ target.

In each simulation, noble gas atoms ( $\mathrm{He}, \mathrm{Ne}$, or Ar) are injected vertically into the tungsten. The initial kinetic energy of the irradiated noble gases are changed from $10 \mathrm{eV}$ to $10 \mathrm{keV}$. The irradiated noble gas atom is collided with $\mathrm{W}$, and loses its kinetic energy. If its kinetic energy becomes less than the threshold energy, i.e., $5 \mathrm{eV}$, the simulation is stopped. The injection position of the noble gas atom was changed randomly at each simulation. The above simulation is repeated $N_{0}=200,000$ times for each tungsten.

\subsection{Simulation Result}

Using this simulation model, we obtain the dependence of the following three physical quantities, i.e., the sputtering yield, the retention range, and the mean penetration depth, on both the injection atom and its kinetic energy. The way to calculate the sputtering yield is as follows. The number of the tungsten atoms $N_{\mathrm{W}}$ which fly away from the W surface is counted. The sputtering yield is given by $N_{\mathrm{W}} / N_{0}$. The retention rate is the ratio between the number of the injection atoms absorbed in the $\mathrm{W}$ target and $N_{0}$. The mean penetration depth is the average depth from the $\mathrm{W}$ surface to the end position of the injected noble gas.

\subsubsection{Sputtering Yield}

First, we calculated the dependence of the sputtering yield on the incident energy of the projectile in the case that the target has the amorphous, (110), (100), or (111) crystalline BCC structure. It is found, from Fig. 2, that the sputtering threshold energies are $100 \mathrm{eV}$, $30 \mathrm{eV}$, and $20 \mathrm{eV}$ for $\mathrm{He}, \mathrm{Ne}$, and Ar, respectively. As the atomic number becomes larger, 


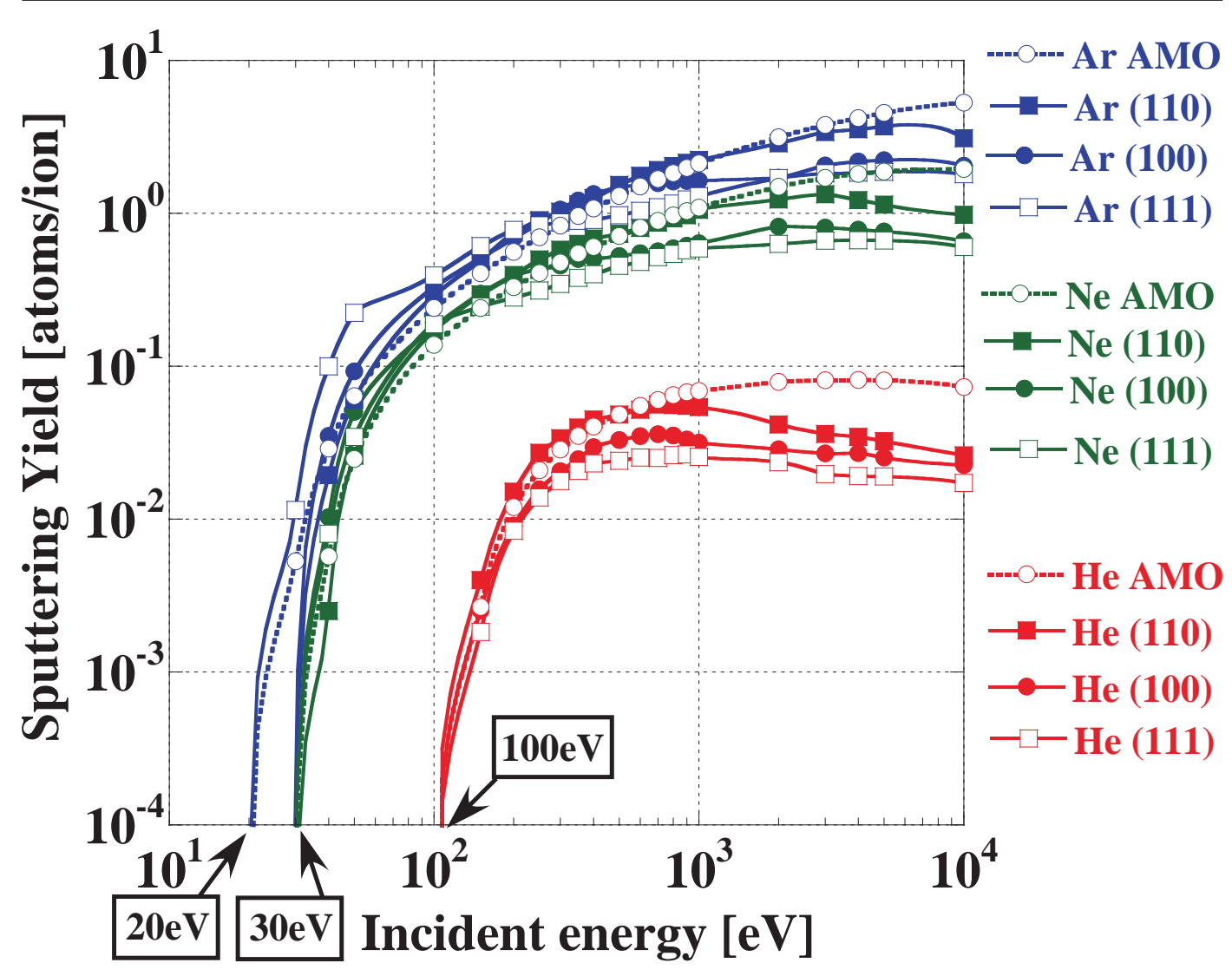

Figure 2: Sputtering yield vs the incident energy of the irradiated noble gas. As the atomic number becomes larger, the sputtering yield increases.

the sputtering yield increases for each incident energy. Moreover, when the incident energy is larger than about $300 \mathrm{eV}, \mathrm{W}$ atoms in the amorphous structure are sputtered most easily in all structures for each incident noble gas. On the other hand, the BCC crystal with (111) surface is most difficult to be sputtered. This property indicates that the binding energy between tungsten atoms in the (111) surface is the larger than the amorphous structure and the crystals with (100) and (110) surfaces.

\subsubsection{Retention Rate}

Next, the retention rate of the noble gases is calculated as shown in Fig. 3. The behavior of the retention rates is complicated in the case that the incident energy is less than several hundred $\mathrm{eV}$, where the channeling effect does not occur (see $\S 2.3 .3$. and Fig. 4). The dependence on the lattice structure becomes clear when the existence of the channeling effect is observed, that is, when the incident energy is larger than about $100 \mathrm{eV}$. It is found that the noble gases are absorbed most by the BCC crystal with (111) surface. However, the amorphous structure has the most difficulty in retaining of the noble gases. 


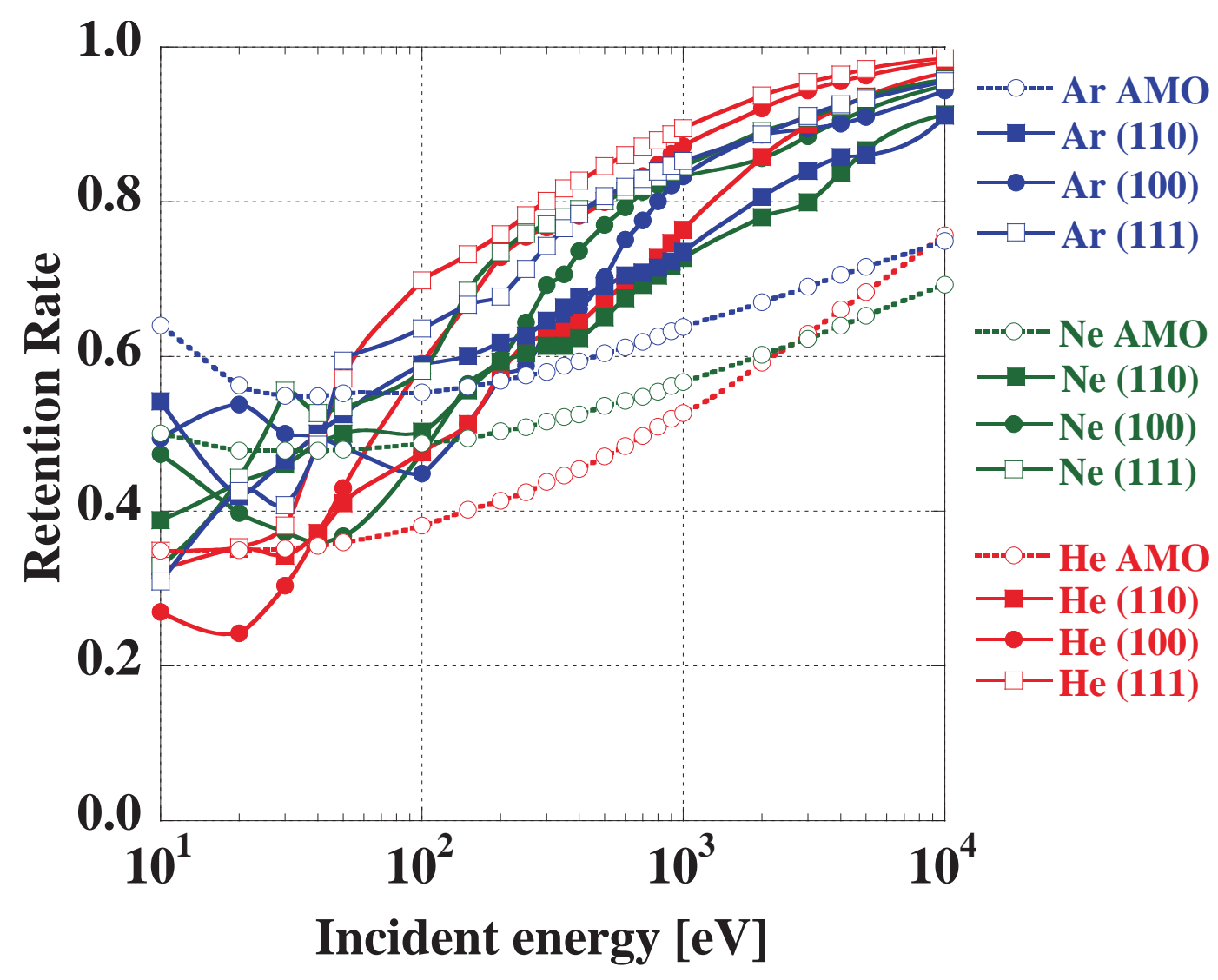

Figure 3: Retention rate vs the incident energy of the irradiated noble gases. The BCC crystal with (111) surface absorbs more noble gases than the other structures. The amorphous structure has the most difficulty in absorbing the gases in all structures. 


\subsubsection{Mean Penetration Depth}

The mean penetration depth is plotted in Fig. 4. This figure shows the channeling effect, that is, when the target material has mono-crystalline structure, the trajectory of the injected atom in the target depends on the orientation of the atom relative to the crystalline axes. The inversion energy $E_{\text {inv }}$ is defined by the incident energy where the mean penetration depth for the BCC crystal becomes larger than that for the amorphous structure. In the caption of Fig. 4 we summarize the values of $E_{\text {inv }}$, which depend on the crystalline structure of the target and the atomic species of the incident gas. Moreover, it is found that the $\mathrm{W}$ crystal with (110) surface is more difficult to generate the channeling effect than the other surfaces, i.e., (100) and (111).

\section{Conclusion}

By the binary collision approximation simulation, we calculated the physical quantities, i.e., sputtering yield, retention rate, and mean penetration depth for each tungsten target with the crystal orientation (110), (100), (111), or the amorphous structure. Thus, it was found that the physical quantities for sputtering phenomena depend strongly on the crystalline orientation of the tungsten surface. On the other hand, by our previous work [8], we have shown that the physical quantities also depend on the roughness of the tungsten surface. Considering the above two results, we propose that it is necessary to consider the structure of the target surface more exactly than the "flat" and amorphous structured surface model which is popular in order to evaluate the endurance of plasma facing material.

\section{Acknowledgement}

This work was supported by JSPS KAKENHI Grant Numbers (15K06650 and 25249132) and the National Institute for Fusion Science (NIFS) Collaboration Research programs (NIFS14KNTS028, NIFS14KNTS043).

\section{References}

[1] S. Takamura, N. Ohno, D. Nishijima, S. Kajita: Formation of Nanostructured Tungsten with Arborescent Shape due to Helium Plasma Irradiation Plasma Fusion Res., 1 (2006) 051 (4 pages).

[2] S. Kajita, T. Saeki, Y. Hirahata, et al.: Development of Nanostructured Black Metal by Self-Growing Helium Bubbles for Optical Application, Jpn. J. Appl. Phys., 50:8S1 (2011), 08JG01 (4 pages).

[3] D. Nishijima, M.J. Baldwin, R.P. Doerner, J. H. Yu: Sputtering Properties of Tungsten ‘fuzzy' Surfaces, J. Nucl. Mater. Supplement, 415:1 (2011), S96-S99. 


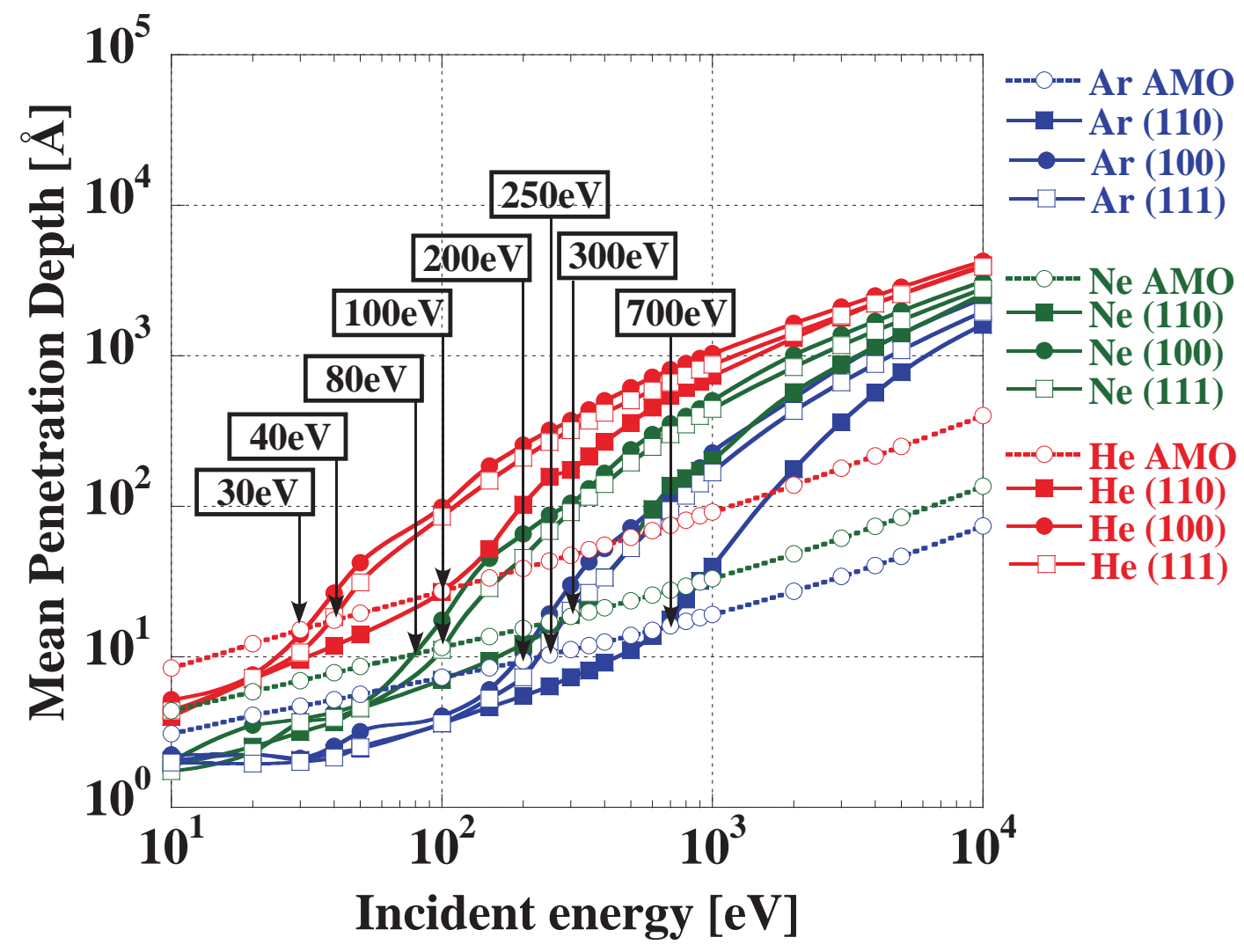

Figure 4: Mean penetration depth vs the incident energy of the irradiated noble gas. The mean penetration depth of BCC lattices with (100) becomes larger than the amorphous structure when the inversion energy $E_{\text {inv }}$ is about $30 \mathrm{eV}$ for $\mathrm{He}$, about $80 \mathrm{eV}$ for $\mathrm{Ne}$ and about $200 \mathrm{eV}$ for Ar, respectively. $E_{\text {inv }}$ for (111) is about $40 \mathrm{eV}$ for $\mathrm{He}$, about $100 \mathrm{eV}$ for $\mathrm{Ne}$ and about $250 \mathrm{eV}$ for Ar, respectively. For the BCC lattice with (110), the inversion of the mean depth occurs in the case of $100 \mathrm{eV}, 300 \mathrm{eV}$, and $700 \mathrm{eV}$ for $\mathrm{He}, \mathrm{Ne}$, and Ar, respectively. Such inversion of the mean penetration depth is caused by the channeling effect. 
[4] A. Takayama, S. Saito, A.M. Ito, T. Kenmotsu, et al.: Extension of Binary-CollisionApproximation-Based Simulation Applicable to Any Structured Target Material, Jpn. J. Appl. Phys., 50:1S1 (2011), 01AB03 (4 pages).

[5] S. Saito, A. Takayama, A.M. Ito, H. Nakamura: How to Combine Binary Collision Approximation and Multi-Body Potential for Molecular Dynamics, Progress in Nuclear Science and Technology, 2 (2011) 44-50.

[6] S. Saito, A. Takayama, A.M. Ito, H. Nakamura: Binary-collision-approximation-based simulation of noble gas irradiation to tungsten materials, J. Nucl. Mater. Supplement, 438 (2013) S895 - S898.

[7] H. Nakamura, S. Saito, A. M. Ito, A. Takayama: Incident energy dependence of reflection rate of gas irradiated on tungsten, 6th International Symposium on Advanced Plasma Science and Its Applications for Nitrides and Nanomaterials/ 7th International Conference on Plasma-Nano Technology E Science (ISPlasma2014/ ICPLANCTS2014), Meijo University, Nagoya, Japan, March 2-6 (2014) 06aP06.

[8] H. Nakamura, S. Saito, A.M. Ito, A. Takayama: Tungsten-surface-structure Dependence of Sputtering Yield for a Noble Gas, Plasma Fusion Res, 11(2016) 2401080 (5 pages). 